\title{
Polymersomes with Aggregation-Induced Emission Based on Amphiphilic Block Copolypeptoids
}

Received 00th January 20xx, Accepted 00th January 20xx DOI: $10.1039 / x 0 x \times 00000 x$

\author{
Xinfeng Tao, ${ }^{\mathrm{a}, \mathrm{b}}$ Hui Chen, ${ }^{\mathrm{b}}$ Sylvain Trépout, ${ }^{\mathrm{e}}$ Jiayu Cen, ${ }^{\mathrm{c} J u n}$ Ling, ${ }^{\mathrm{c}}$ Min-Hui Li*b,d
}

\begin{abstract}
Biocompatible polymersomes are prepared from amphiphilic block copolypeptoids with aggregation-induced emission, where the hydrophobic block P(TPE-NAG) is a tetraphenylethylene (TPE)-modified poly( $N$-allylglycine) and the hydrophilic block is polysarcosine. These nanoparticles are non-cytotoxic and show strong fluorescence emission in aqueous solution.
\end{abstract}

Nanoparticles composed of amphiphilic copolymers have attracted extensive attention in the past decades due to their ordered nanostructures with various morphologies, including spherical micelles, cylindrical micelles and vesicles, etc., which can be potentially used in drug delivery and bioimaging systems. ${ }^{1}$ Among them, polymer vesicles, also known as polymersomes, are cell-mimicking hollow spheres with a polymer bilayer membrane enclosing an aqueous volume in its interior. They are more stable and robust than lipid vesicles (liposomes) and have high encapsulation capacity for both hydrophilic and hydrophobic molecules. ${ }^{2,3}$

Polypeptoids are a class of poly(amino acid)s suitable for the polymersome preparation with the advantages of excellent biocompatibility, degradability, and low cytotoxicity. ${ }^{4}$ Polypeptoids have a protein-mimicking structure with $N$ substituted glycine as repeating units but lack intramolecular and intermolecular hydrogen bonding along the amino acid

\footnotetext{
a. Shanghai Key Laboratory of Advanced Polymeric Materials, School of Materials Science and Engineering, East China University of Science and Technology, Shanghai 200237, China.

b. Chimie ParisTech, PSL Université Paris, CNRS, Institut de Recherche de Chimie Paris, UMR8247, 11 rue Pierre et Marie Curie, 75005 Paris, France.

MOE Key Laboratory of Macromolecular Synthesis and Functionalization, Department of Polymer Science and Engineering, Zhejiang University, Hangzhou 310027, China.

d. Beijing Advanced Innovation Center for Soft Matter Science and Engineering, Beijing University of Chemical Technology, 15 North Third Ring Road, Chaoyang District, 100029 Beijing, China.

e. Institut Curie, PSL Université Paris, INSERM U1196 and CNRS UMR9187, 91405 Orsay cedex, France.

† Footnotes relating to the title and/or authors should appear here.

Electronic Supplementary Information (ESI) available: [details of any supplementary information available should be included here]. See DOI: 10.1039/x0xx00000x
}

backbone. Therefore, polypeptoids show better solubility, thermal processability, cell permeability and proteolytic stability compared to polypeptides. ${ }^{4}$ Polypeptoids are usually synthesized by ring-opening polymerization (ROP) of $\mathrm{N}$ substituted glycine $\mathrm{N}$-carboxyanhydride (NNCA). ${ }^{4}$ Recently, we developed an efficient alternative way to synthesize welldefined polypeptoids using of a more stable monomer $\mathrm{N}$ substituted glycine $N$-thiocarboxyanhydride (NNTA). ${ }^{5}$

Nanoparticles based on polypeptoids have been widely investigated. ${ }^{6}$ Luxenhofer and co-worker prepared worm-like micelles and polymersomes by using amphiphilic block copolypeptoids containing hydrophilic polysarcosine (PSar) block and hydrophobic polypeptoid block with butyl, pentyl, benzyl or phenethyl side groups. ${ }^{7}$ We reported previously that PSar-block-poly( $\varepsilon$-caprolactone)-block-PSar (PSar-b-PCL-b-PSar) formed unilamellar sheets, worm-like cylinders, and multilamellar polymersomes under different conditions. ${ }^{8}$ Spherical micelles formed in water by poly( $N$-ethylglycine)block-poly[( $N$-propargylglycine)-r-( $N$-decylglycine)] (PNEG- $b$ P(NPgG-r-NDG)) were studied by Zhang et al. ${ }^{9}$ They used the hydrophobic core of micelles to encapsulate Doxorubicin (DOX) for further application of controlled release of DOX. ${ }^{9}$ Sun et al. synthesized different PEG-block-polypeptoid which selfassembled into two-dimensional nanosheets. ${ }^{10,11}$

All the above reported nanoparticles are based on polypeptoids with normal aliphatic or aromatic side chains. Nevertheless, functional side chains are often expected in various applications, for example, for the preparation of stimuliresponsive nanoparticles in controlled release or of fluorescent nanoparticles in bioimaging. In a recent paper, we developed oxidation-responsive polymersomes based on copolypeptoids, poly( $N$-3-(methylthio)propyl glycine)-block-PSar (PMeSPG- $b$ PSar), containing thioether side-chains on PMeSPG. ${ }^{12}$ However, this method has some limitation, i.e., we need to develop a specific route of synthesis for each functional monomer, which is tedious and time-consuming. In this study, we propose to use a functionalizable poly( $N$-allylglycine) (PNAG) as hydrophobic block, which is an excellent platform for modification through 


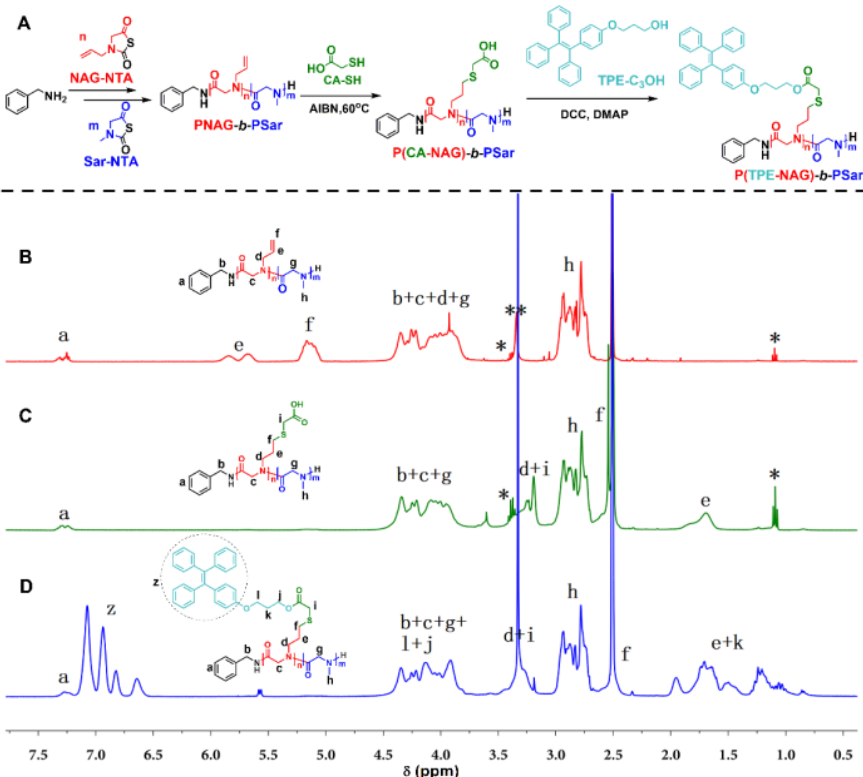

Fig 1. (A) Synthesis of PNAG- $b$-PSar through block copolymerization of NAG-NTA and SarNTA and its postpolymerization modification by reacting with mercaptoacetic acid and TPE- $\mathrm{C}_{3} \mathrm{OH}$ successively to produce $\mathrm{P}(\mathrm{TPE}-\mathrm{NAG})-b$-PSar. ${ }^{1} \mathrm{H}$ NMR spectra of $\mathrm{PNAG}_{15}-b$ PSar $_{54}$ (B), P(CA-NAG) $)_{15}-b-$ PSar $_{54}$ (C) and P(TPE-NAG) ${ }_{15}-b-$ PSar $_{54}$ (D) in DMSO- $d_{6}$. (* diethyl ether; ${ }^{* *}$ : water)

thiol-ene reaction to get functional polypeptoids. ${ }^{13}$ PNAG- $b$ PSar was synthesized using benzylamine-initiated ROP of $\mathrm{N}$ allylglycine NTA (NAG-NTA) and sarcosine NTA (Sar-NTA). To highlight their ability to be functionalized in side-chains and to self-assemble into functional polymersomes, we attached the tetraphenylethylene (TPE), ${ }^{14-18}$ the emblematic luminogen with aggregation-induced emission (AIE), to PNAG side chains through postpolymerization modification (Fig. 1A). Polymersomes and micelles with AIE properties were obtained by using nanoprecipitation method. Cytotoxicity test showed good biocompatibility of these nanoparticles. We believe these AIE polymersomes based on polypeptoids will have potential application in bio-imaging and drug delivery.

We first synthesized Sar-NTA and the novel monomer NAGNTA through a phosgene-free method (Fig. S1-S6, ESI). ${ }^{5}$ Homopolymerization of NAG-NTA initiated by benzylamine in acetonitrile at $60{ }^{\circ} \mathrm{C}$ was then studied (Table S1, ESI). The wellcontrolled characteristic of NAG-NTA polymerization based on single-site initiation by benzylamine was supported by MALDIToF mass spectra, ${ }^{1} \mathrm{H}$ NMR spectra and SEC traces (see ESI and Fig. S8-S10 for details). PNAG can be modified easily through thiol-ene radical addition reaction with mercaptan, e.g. mercaptoacetic acid, mercaptoethanol, and benzyl mercaptan (Scheme S5, ESI). The ${ }^{1} \mathrm{H}$ NMR spectra (Fig. S9, ESI) indicated the quantitative transformation of allyl groups to the selected functional groups.

PNAG- $b$-PSar with three different molecular weights were then synthesized by sequential feeding of different amounts of NAG-NTA and Sar-NTA (Fig. 1A). The successful synthesis of PNAG- $b$-PSar was proved by ${ }^{1} \mathrm{H}$ NMR spectra and SEC traces (see Fig. 1B, Fig. S11 and discussion in ESI). The compositions of the three block copolymers were $\mathrm{PNAG}_{10}-b$ - $\mathrm{PSar}_{53}, \mathrm{PNAG}_{15}-b$ - $\mathrm{PSar}_{54}$ and $\mathrm{PNAG}_{25}-b$-PSar 46 as calculated from NMR data.
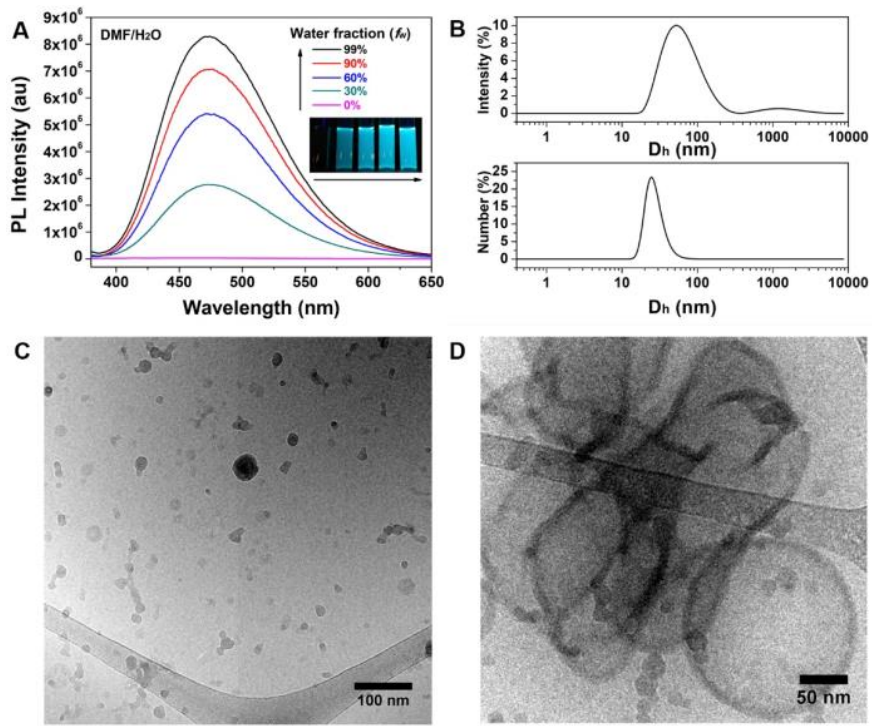

Fig. 2. (A) Photo-luminescence (PL) spectra of $P(T P E-N A G)_{25}-b-P S a r_{46}$ in $D M F / H_{2} O$ mixtures with different water fractions (concentration: $2.2 \times 10^{-5} \mathrm{M}, 0.4 \mathrm{mg} / \mathrm{mL}$; excitation wavelength: $370 \mathrm{~nm}$ ). The inserted photographs in (A) represent samples with different water fractions under UV light of $365 \mathrm{~nm}$. (B) DLS profiles (Z-average with intensity and number average) of aggregates of $\mathrm{P}(\mathrm{TPE}-\mathrm{NAG})_{25}-b$-PSar ${ }_{46}$ in $\mathrm{DMF} / \mathrm{H}_{2} \mathrm{O}$ mixture with water fraction at $99 \%$. (C) and (D) cryo-EM images of aggregates of $P(T P E-$ $\mathrm{NAG})_{25}-b-\mathrm{PSar}_{46}$ in $\mathrm{DMF} / \mathrm{H}_{2} \mathrm{O}$ mixture with water fraction at $99 \%$.

AIE luminogens were attached to the block copolymers by two-step reactions (Fig. 1A). PNAG- $b$-PSar was first reacted with mercaptoacetic acid (CA-SH) to get carboxylic acidfunctionalized P(CA-NAG)-b-PSar (Fig. 1C), which was then reacted with 3-(4-(1,2,2-triphenylvinyl)phenoxy)propan-1-ol (TPE- $\mathrm{C}_{3} \mathrm{OH}$ ) (Scheme S4 and Fig. S7, ESI) to get the AIE block copolymers $\mathrm{P}(\mathrm{TPE}-\mathrm{NAG})-b$-PSar. The final structures of P(TPENAG)- $b$-PSar were confirmed by ${ }^{1} \mathrm{H}$ NMR spectra (Fig. 1D and Fig. S12) and SEC traces (Fig. S13). Their molecular weights $(\mathrm{MW})$ and distributions $(\Theta)$ are summarized in Table S2. The hydrophilic weight ratios $\left(f_{\mathrm{PSar}}\right)$ of $\mathrm{P}(\mathrm{TPE}-\mathrm{NAG})-b$-PSar are $18 \%$, $30 \%$ and $38 \%$, respectively.

P(TPE-NAG $)_{25}-b$-PSar 46 was chosen as an example to study the AIE properties of copolypeptoids. We found it exhibited obvious AIE fluorescence when dispersed in water owing to the restriction of intramolecular rotation (RIM) of TPE moieties in the aggregated state (Fig. 2A and discussion in ESI). ${ }^{18}$ Moreover, we were also interested in the morphology of aggregates formed in this experiment. The dispersion in $\mathrm{DMF} / \mathrm{H}_{2} \mathrm{O}$ mixture with $f_{\mathrm{w}}=99 \%$ was selected for DLS and cryo-EM analysis. Fig. $2 \mathrm{~B}$ shows the size distribution of aggregates weighed by scattering intensity and by number. There are two populations of nanoparticles, where the smaller one at about $56 \mathrm{~nm}$ of average hydrodynamic diameter is predominant and a few of big particles with diameters $>1000 \mathrm{~nm}$ co-exist. Fig. 2C and D show the nanostructures observed by cryo-EM, which confirm the results of DLS. Many nanoparticles with diameters $<50 \mathrm{~nm}$ were observed. Note that hydrophilic chains located on the membrane coronas (exterior and interior sides) of the polymersomes are flexible and swollen in water, which do not constitute enough density contrast, relative to vitreous ice at the sample grid, to be visible in cryo-EM. ${ }^{19}$ Therefore, it is normal that the sizes measured by cryo-EM are smaller than the 
hydrodynamic diameters. Meanwhile, a few of vesicles (Fig. 2D, Fig. S15) are also observed, and some of them have very big
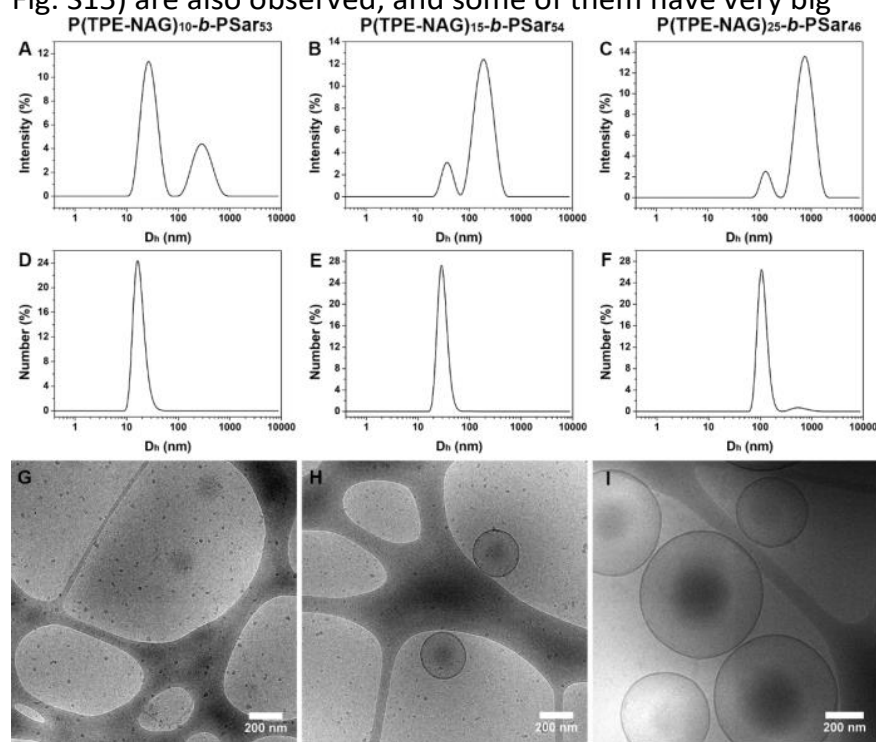

Fig. 3. DLS profiles (Z-average with intensity $A-C$ and number average $D-F$ ) and cryo-EM images of nanoparticles in water formed by $\mathrm{P}(\mathrm{TPE}-\mathrm{NAG})_{10}-b$-PSar ${ }_{53}\left(f_{\mathrm{Psar}}=38 \%\right)(\mathrm{A}, \mathrm{D}$ and G), P(TPE-NAG $)_{15}-b$-PSar $54\left(f_{\text {Psar }}=30 \%\right)(B, E$ and H $)$ and P(TPE-NAG $)_{25}-b-$ PSar $_{46}\left(f_{\text {Psar }}=18 \%\right)$ (C, $\mathrm{F}$ and $\mathrm{I}$ ). The dark densities present at the center of the polymersomes are caused by the scattering of electrons due to the inherent thickness of the polymersome spheres.

size, which correspond effectively to the second population of particles in DLS curves. These observations showed that the copolypeptoid P(TPE-NAG) 25 - $b$-PSar ${ }_{46}$ had the tendency to form vesicular structures. However, well defined polymersomes were not obtained probably because of the quick addition of large quantity of water.

Then, we used a nanoprecipitation method with DMF/water as co-solvents and a slow water addition process to selfassemble P(TPE-NAG)- $b$-PSar in a more controlled way. Typically, P(TPE-NAG)- $b$-PSar was first dissolved in $1 \mathrm{~mL}$ DMF with a concentration of $2 \mathrm{mg} / \mathrm{mL}$, and deionized water was added then very slowly $(2.5 \mu \mathrm{L} / \mathrm{min})$ with slight shaking. The slow rate of water addition could avoid the early freezing of nanostructure and keep the self-assembly dynamic as long as possible. When the water content was increased to around $70 \%$ in volume, the dispersions were dialyzed against deionized water to remove DMF and get P(TPE-NAG)- $b$-PSar selfassemblies in pure water. Table S2 summarizes hydrodynamic diameters of all nanoparticles and membrane thicknesses of the polymersomes. For $\mathrm{P}(\mathrm{TPE}-\mathrm{NAG})_{10}-b$-PSar ${ }_{53}\left(f_{\mathrm{Psar}}=38 \%\right)$, micelles with a diameter around $26 \mathrm{~nm}$ are mainly observed by cryo-EM (Fig. 3G, Fig. S16) and TEM (Fig. S17). DLS intensity profile shows a bimodal distribution (Fig. 3A) with the hydrodynamic diameters of $29 \mathrm{~nm}$ and $312 \mathrm{~nm}$, respectively. When there are several populations of nanoparticles, it is difficult to quantify the statistics with only one kind of size profile in DLS because the intensity profile privileges the big particles and the number profile privileges the small particles. Therefore, both intensityweighed and number-weighed DLS curves are given here (Fig. $3 \mathrm{~A}$ and $3 \mathrm{D}$ ). The smaller one corresponds to the micelles, while the larger one (very few in number as shown in number profile of DLS) may attribute to a few of aggregations (clusters) of micelles (Fig. 3G and Fig. S16). When the $f_{\text {Psar }}$ decreases to 30\% (P(TPE-NAG) ${ }_{15}-b$-PSar $\left.{ }_{54}\right)$, both micelles and polymersomes were observed by cryo-EM (Fig. $3 \mathrm{H}$ and Fig. S18) and TEM (Fig. S19), whose diameters are $26 \mathrm{~nm}$ and $357 \mathrm{~nm}$, respectively. DLS profile of P(TPE-NAG) ${ }_{15}-b$-PSar ${ }_{54}$ nanoparticles are double peaks with the hydrodynamic diameters of $38 \mathrm{~nm}$ and $203 \mathrm{~nm}$ (Fig. $3 \mathrm{~B})$, which is consistent with the TEM results. Note that the polymersomes were minor population as shown in DLS number profile and connected or fused polymersomes were observed in some cases (Fig. S18). In the case of $\mathrm{PNAG}_{25}-b$-PSar $46\left(f_{\mathrm{Psar}}\right.$ $=18 \%$ ), only polymersomes are formed according to cryo-EM images (Fig. 3l and Fig. S20), with two distributions of sizes: one around a Z-average diameter of $804 \mathrm{~nm}$, and another around a Z-average diameter of $136 \mathrm{~nm}$ as measured by DLS (Fig. 3C and $3 F)$. As for the sizes and size distributions, the results obtained from cryo-EM and DLS (Table S2) are coherent to some extent.

With the increase of the hydrophobic P(TPE-NAG) chain length and the decrease of the hydrophilic ratio $f_{\text {Psar }}$ (here the length of hydrophilic part PSar is similar for three copolypeptoids), the nanostructures transform from micelles to vesicles and their average sizes become larger. These tendencies can be empirically explained by the increase of the packing parameter $p=v / a l$ ( $v$ is the hydrophobic volume, $a$ is the optimal interfacial area and $l$ is the length of the hydrophobic block normal to the interface) when increasing hydrophobic block MW, as already reported previously. ${ }^{15,} 20,21$

According to the cryo-EM images, the thickness of the hydrophobic part of membrane is measured as to be $8 \pm 1 \mathrm{~nm}$ and $10 \pm 1 \mathrm{~nm}$ for P(TPE-NAG) ${ }_{15}-b$-PSar ${ }_{54}$ and P(TPE-NAG) ${ }_{25}-b$ $\mathrm{PSar}_{46}$ polymersomes, respectively. It is easy to understand that the longer the hydrophobic block leads to the thicker membrane. By using the MM2 energy minimization with the Chem3D software, the contour lengths of the hydrophobic part $P(\text { TPE-NAG })_{15}$ and P(TPE-NAG $)_{25}$ are about $4.4 \mathrm{~nm}$ and $7.3 \mathrm{~nm}$, respectively. Consequently, a perfectly-ordered tail-to-tail bilayer should have a membrane thickness of $8.8 \mathrm{~nm}$ and 14.3 $\mathrm{nm}$, respectively. The membrane thickness measured by cryo$E M$ is lower than the double lengths of two extended P(TPENAG) chains. Therefore, the obtained polymersomes should be unilamellar vesicles, in which P(TPE-NAG) chains might not be totally extended or might be interdigitated between the two leaflets.

Then, we investigated the fluorescence of these well-defined nanostructures formed by P(TPE-NAG)- $b$-PSar. The DMF solution of $\mathrm{P}(\mathrm{TPE}-\mathrm{NAG})-b$-PSar is non-emissive while the aqueous solution of $\mathrm{P}$ (TPE-NAG)- $b$-PSar micelles and vesicles shows strong emission under $365 \mathrm{~nm}$ UV lamp (Fig. 4A) due to the RIM of TPE moieties in the core of polymer micelles and of polymersome membrane as expected. The emission spectra of nanoparticles of P(TPE-NAG) ${ }_{10}-b$-PSar ${ }_{53}, \mathrm{P}(\mathrm{TPE}-\mathrm{NAG})_{15}-b$-PSar $_{54}$ and $\mathrm{P}(\mathrm{TPE}-\mathrm{NAG})_{25}-b$-PSar ${ }_{46}$ are shown in Fig. $4 \mathrm{~B}$ with maximal emission wavelengths at $478 \mathrm{~nm}, 474 \mathrm{~nm}$ and $471 \mathrm{~nm}$, respectively, the excitation wavelength being set as $370 \mathrm{~nm}$ that corresponded to the maximal wavelength in the excitation spectrum (Fig. S21). Rather high quantum yields were measured as $26 \%, 29 \%$ and $34 \%$ for P(TPE-NAG) ${ }_{10}-b$-PSar ${ }_{53}, \mathrm{P}(\mathrm{TPE}-\mathrm{NAG})_{15}-$ $b$-PSar 54 and $\mathrm{P}(\mathrm{TPE}-\mathrm{NAG})_{25}-b$-PSar $_{46}$ nanoparticles, respectively, 
using the integrating sphere method. The fluorescence intensity and quantum yield increase following the morphology evolution

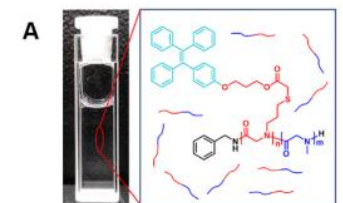
DMF solution

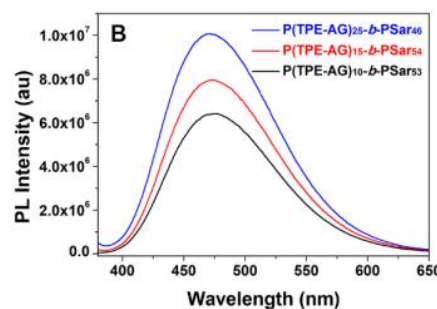

Nanoprecipitation

Self-Assembling

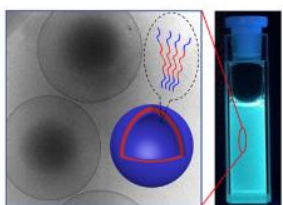

Aqueous solution

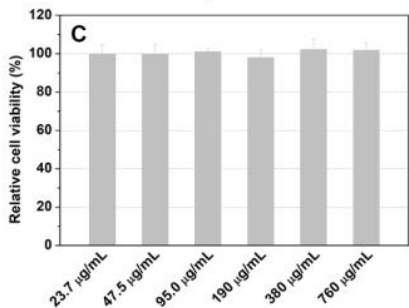

Fig. 4. (A) Schematic diagram of self-assembly of $P(T P E-N A G)-b-P S a r$ in aqueous solution and the AIE phenomenon of the polymersome dispersion. (B) PL spectra of different nanoparticles (concentration: $0.4 \mathrm{mg} / \mathrm{mL}$; excitation wavelength: $370 \mathrm{~nm}$ ). (C) Relative cell viability of $\mathrm{P}(\mathrm{TPE}-\mathrm{NAG})_{25}-b-\mathrm{PSar}_{46}$ polymersomes for human vein endothelial cells after $24 \mathrm{~h}$ incubation at a concentration of $23.7 \mu \mathrm{g} / \mathrm{mL}, 47.5 \mu \mathrm{g} / \mathrm{mL}, 95.0 \mu \mathrm{g} / \mathrm{mL}, 190$ $\mu \mathrm{g} / \mathrm{mL}, 380 \mu \mathrm{g} / \mathrm{mL}$ and $760 \mu \mathrm{g} / \mathrm{mL}$, respectively.

from micelles, micelles/polymersomes to polymersomes, which is attributed to the increasing packing density of polymer blocks within the nanoparticles and the increasing confinement of the AlEgens. ${ }^{14}$

Finally, the biocompatibility of the nanoparticles was assessed by using MTT cell viability assay. ${ }^{22}$ Human vein endothelial cells were incubated for $24 \mathrm{~h}$ with P(TPE-NAG)- $b$ PSar nanoparticles at different concentrations. All P(TPE-NAG)$b$-PSar micelles and polymersomes studied are non-cytotoxic (Fig. 4C, Fig. S22 and S23), indicating their excellent biocompatibility. The negligible cytotoxicity of P(TPE-NAG)- $b$ PSar polymersomes makes possible their applications in biorelated domains such as bio-imaging and drug delivery.

In summary, $\mathrm{N}$-allylglycine NTA was synthesized for the first time, whose corresponding polymer PNAG is an excellent platform for post-modification to produce different functional polypeptoids. Well-controlled polymerization of NAG-NTA was achieved by using primary amine as the initiator, and PNAGs with narrow distributions were produced. A series of diblock copolypeptoids PNAG- $b$-PSar with different chain lengths were synthesized by sequential feeding of NAG-NTA and Sar-NTA. TPE-functionalized P(TPE-NAG) ${ }_{10}-b$-PSar $53\left(f_{\text {Psar }}=38 \%\right), \mathrm{P}(\mathrm{TPE}-$ $\mathrm{NAG})_{15}-b-\mathrm{PSar}_{54}\left(f_{\mathrm{Psar}}=30 \%\right)$ and P(TPE-NAG $)_{25}-b-\mathrm{PSar}_{46}\left(f_{\mathrm{Psar}}=\right.$ $18 \%)$ were prepared by post-polymerization reaction. Selfassembling was performed by nanoprecipitation method using DMF/water as co-solvents. Micelles, micelles/polymersomes and polymersomes were obtained for P(TPE-NAG) ${ }_{10}-b$-PSar ${ }_{53}$, $\mathrm{P}(\mathrm{TPE}-\mathrm{NAG})_{15}-b$-PSar ${ }_{54}$ and $\mathrm{P}(\mathrm{TPE}-\mathrm{NAG})_{25}-b$-PSar $_{46}$, respectively. All the nanoparticles show strong AIE fluorescence in aqueous solution. These AIE polymer micelles and AIE polymersomes made of block copolypeptoids are non-cytotoxic, which guarantee their safety in potential applications in bio-related field.

Financial supports from French National Research Agency (ANR-16-CE29-0028) and National Natural Science Foundation of China (21674091) are acknowledged. We thank Prof. Ben Zhong Tang (Hong Kong University of Science and Technology) for fruitful discussions. We thank Prof. Zhengwei Mao (Zhejiang University) for cytotoxicity analysis, and Dr. Chao Deng (Zhejiang University) for quantum yield measurement. The authors thank the PICT-Ibisa for providing access to the cryo-EM facility at Institut Curie in Orsay.

\section{Conflicts of interest}

There are no conflicts to declare.

\section{Notes and references}

1 Y. Mai and A. Eisenberg, Chem. Soc. Rev., 2012, 41, 59695985.

2 Y. Deng, J. Ling and M.-H. Li, Nanoscale, 2018, 10, 6781-6800.

3 E. Rideau, R. Dimova, P. Schwille, F. R. Wurm and K. Landfester, Chem. Soc. Rev., 2018, 47, 8572-8610.

4 N. Gangloff, J. Ulbricht, T. Lorson, H. Schlaad and R. Luxenhofer, Chem. Rev., 2016, 116, 1753-1802.

5 X. F. Tao, M.-H. Li and J. Ling, Eur. Polym. J., 2018, 109, 2642.

6 A. Birke, J. Ling and M. Barz, Prog. Polym. Sci., 2018, 81, 163208.

7 C. Fetsch, J. Gaitzsch, L. Messager, G. Battaglia and R. Luxenhofer, Sci. Rep., 2016, 6, 33491.

8 Y. W. Deng, T. Zou, X. F. Tao, V. Semetey, S. Trepout, S. Marco, J. Ling and M. H. Li, Biomacromolecules, 2015, 16, 3265-3274.

9 A. Li and D. H. Zhang, Biomacromolecules, 2016, 17, 852-861.

10 Z. Shi, Y. Wei, C. Zhu, J. Sun and Z. Li, Macromolecules, 2018, $51,6344-6351$.

11 Y. Wei, J. Tian, Z. Zhang, C. Zhu, J. Sun and Z. Li, Macromolecules, 2019, 52, 1546-1556.

12 Y. W. Deng, H. Chen, X. F. Tao, F. Y. Cao, S. Trépout, J. Ling and M.-H. Li, Biomacromolecules, 2019, 20, 3435-3444.

13 J. W. Robinson and H. Schlaad, Chem. Commun., 2012, 48, 7835-7837.

14 M. Huo, Q. Q. Ye, H. L. Che, X. S. Wang, Y. Wei and J. Y. Yuan, Macromolecules, 2017, 50, 1126-1133.

15 N. Zhang, H. Chen, Y. Fan, L. Zhou, S. Trepout, J. Guo and M.H. Li, ACS Nano, 2018, 12, 4025-4035.

16 D. Zhang, Y. Fan, H. Chen, S. Trépout and M.-H. Li, Angew. Chem., Int. Ed., 2019, 58, 10260-10265.

17 Q. Liu, Q. Xia, S. Wang, B. S. Li and B. Z. Tang, J. Mater. Chem. C, 2018, 6, 4807-4816.

18 S. Liu, Y. Cheng, H. Zhang, Z. Qiu, R. T. K. Kwok, J. W. Y. Lam and B. Z. Tang, Angew. Chem., Int. Ed., 2018, 57, 6274-6278.

19 G. H. Michler, Electron Microscopy of Polymers, Springer, Berlin, 2008.

20 A. Blanazs, S. P. Armes and A. J. Ryan, Macromol. Rapid Commun., 2009, 30, 267-277.

21 L. Jia, P.-A. Albouy, A. Di Cicco, A. Cao and M.-H. Li, Polymer, 2011, 52, 2565-2575.

22 G. Yu, S. Yu, M. L. Saha, J. Zhou, T. R. Cook, B. C. Yung, J. Chen, Z. Mao, F. Zhang, Z. Zhou, Y. Liu, L. Shao, S. Wang, C. Gao, F. Huang, P. J. Stang and X. Chen, Nat. Commun., 2018, 9, 4335 . 